\title{
Synchronous papillary urothelial carcinoma of the bladder and squamous cell carcinoma with sarcomatoid differentiation: A case report
}

\author{
MAHIZER YALDIZ ${ }^{1}$, NUR CIHAN COSANSU ${ }^{1}$, BERNA SOLAK ${ }^{1}$, \\ ZEYNEP KAHYAOGLU $^{2}$ and MUSTAFA TEOMAN ERDEM ${ }^{1}$ \\ Departments of ${ }^{1}$ Dermatology and ${ }^{2}$ Medical Pathology, Sakarya University Training and Research Hospital, \\ Adapazari, 54100 Sakarya, Turkey
}

Received April 2,2015; Accepted May 19, 2016

DOI: $10.3892 / \mathrm{ol} .2016 .5064$

\begin{abstract}
The incidence rate of multiple primary tumors is $37 \%$ in all types of cancer. A patient diagnosed with primary cancer is 1.29 times more likely to develop an additional primary cancer when compared with the general population. Furthermore, in patients diagnosed with primary cancer, the possibility of a secondary malignancy in the same or different organ is increased. Following the identification of a secondary tumor, the risk of relapse or metastasis must be considered. The present study reports the case of a 76-year-old man who was admitted to Sakarya University Training and Research Hospital (Sakarya, Turkey) with swelling of the head, which had been apparent for 15 days. An excisional biopsy of the temporal region was performed and was used to diagnose the patient with synchronous squamous cell carcinoma with sarcomatoid differentiation of the scalp. The patient was referred to the Department of Plastic Surgery (Sakarya University Training and Research Hospital) for resection; however, he refused treatment and was subsequently discharged. To the best of our knowledge, this patient represents the first case of synchronous skin malignancy and urothelial carcinoma of the bladder to be reported in the literature.
\end{abstract}

\section{Introduction}

Multiple primary cancers (MPCs) occur at a rate of $37 \%$ in all types of cancer (1). A patient with cancer is 1.29 times more likely to develop an additional primary cancer when compared with the risk of cancer in the general population (1). Synchronous tumors are defined as primary tumors that are

Correspondence to: Dr Mahizer Yaldiz, Department of Dermatology, Sakarya University Training and Research Hospital, 195 Saglik Sokak, Adapazari, 54100 Sakarya, Turkey

E-mail: drcanyaldiz@yahoo.com

Key words: synchronous tumors, papillary urothelial carcinoma, squamous cell carcinoma diagnosed at the same time or within 6 months of each other, while primary tumors diagnosed 6 months after the initial primary tumor are defined as secondary or metachronous cancer (2). Secondary primary tumors most commonly occur in the lungs $(27.6 \%)$, head and neck (20.3\%), and urinary system (10.4\%) (3).

A genetic predisposition, which involves mutations in genes that have been shown to be recurrently mutated in specific cancers, has been postulated as a cause for MPC $(4,5)$. Other possibilities include the accumulation of free radicals, mistakes in DNA replication, and the reduced function of lipid-laden macrophages leading to impaired host immune surveillance (4). The increased detection of MPC and potentially synchronous tumors may be attributed to the increased use of advanced diagnostic techniques $(4,6)$. At present, there is no consensus on treatment recommendations for synchronous tumors. The treatment of synchronous tumors is dependent on the tumor's histological subtype, location, and stage (7). The surgical preference is for resection of both tumors simultaneously, whilst modification of behavioral risk factors may prevent the occurrence of MPCs (4).

In the present study, a rare case of synchronous bladder cancer and squamous cell carcinoma with sarcomatoid differentiation of the scalp was reported.

\section{Case report}

A 76-year-old man was admitted to Sakarya University Training and Research Hospital (Sakarya, Turkey) in March 2014 with swelling of the head, which had been apparent for 15 days. The patient had been diagnosed with papillary urothelial carcinoma at the Urology Department of Sakarya University Training and Research Hospital 3 months previously (December 2013). The patient had a history of smoking. Dermatological examination of the right temporal and occipital regions identified three erythematous indurated nodules, which were hard and painful on palpation (Fig. 1). Laboratory examination revealed leukocytosis [white blood cell count, $42.6 \mathrm{~K} / \mu 1$ (normal range, 4.6-10.2 K/ $\mu \mathrm{l}$ )], anemia [hemoglobin count, $11.8 \mathrm{~g} / \mathrm{dl}$ (normal range, 12.2-18.1 g/dl)], neutrophilia [neutrophil count, $36.6 \mathrm{~K} / \mu \mathrm{l}$ (normal range, 2.0-6.9 K/ $/ \mu \mathrm{l}$ )], abnormal renal 


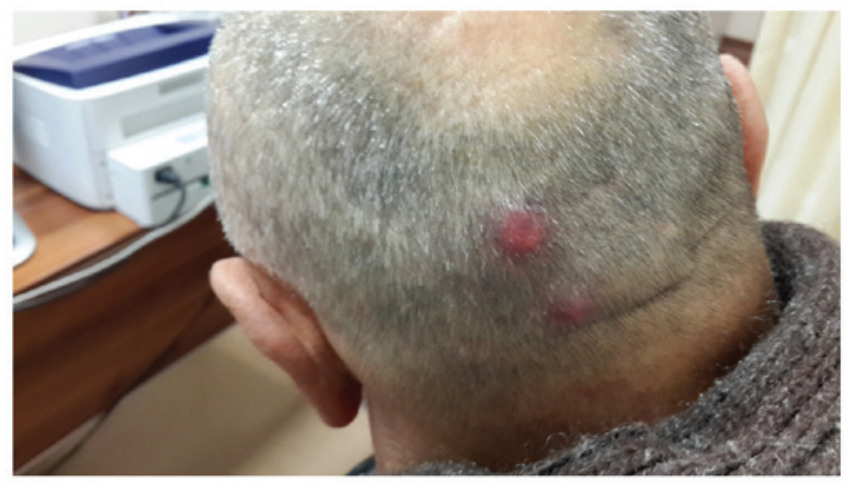

Figure 1. Dermatological examination of the right temporal and occipital regions revealed three painful, hard and indurated erythematous nodules.

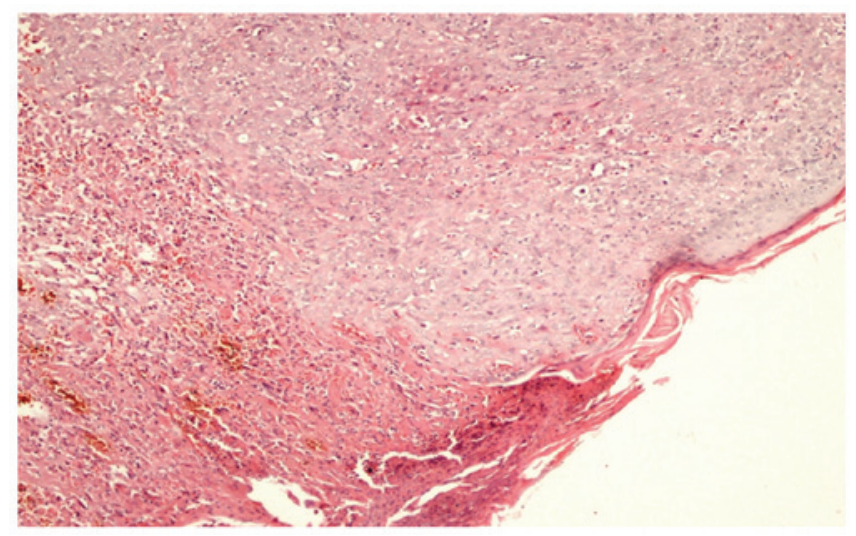

Figure 2. A histological analysis of the right temporal region biopsy showed ulceration of the surface epidermis, dermis and epidermis, and infiltrative tumoral tissue (stain, hematoxylin and eosin; magnification, x100).

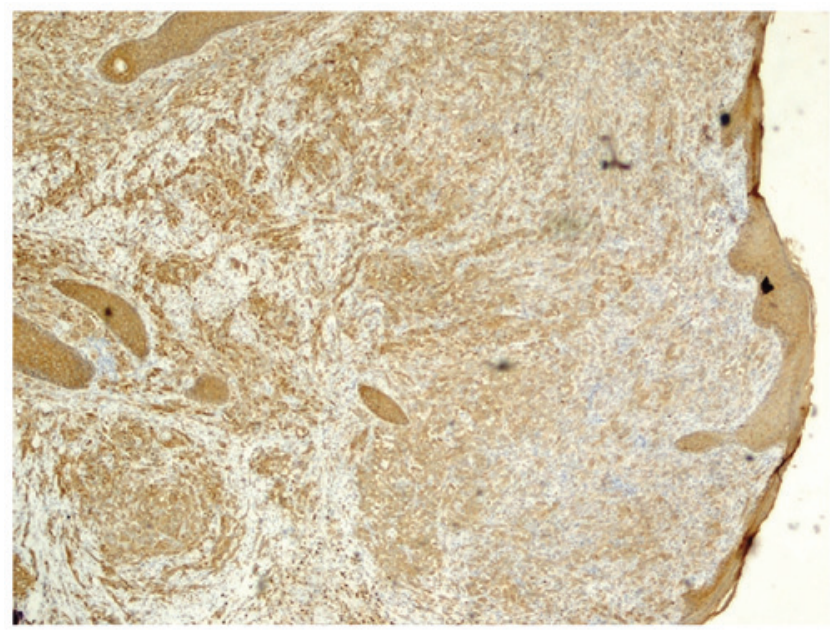

Figure 3. Immunohistochemical staining of tumoral tissue revealed strong, diffuse staining with high molecular weight cytokeratin (magnification, $\mathrm{x} 40$ ).

function [creatine, $2.8 \mathrm{mg} / \mathrm{dl}$ (normal range, $0.7-1.2 \mathrm{mg} / \mathrm{dl}$ ); uric acid, $12.8 \mathrm{mg} / \mathrm{dl}$ (normal range, 3.4-7.0 mg/dl)], and electrolyte imbalances $\left[\mathrm{K}^{+}, 7.5 \mathrm{mmol} / 1\right.$ (normal range, 3.5-5.1 $\mathrm{mmol} / \mathrm{l}$ ); $\mathrm{Ca}^{2+}, 11.4 \mathrm{mg} / \mathrm{dl}$ (normal range, 8.8-10.2 mg/dl)].

An excisional biopsy of the temporal region was performed, and the biopsy specimen was fixed in $10 \%$ formalin

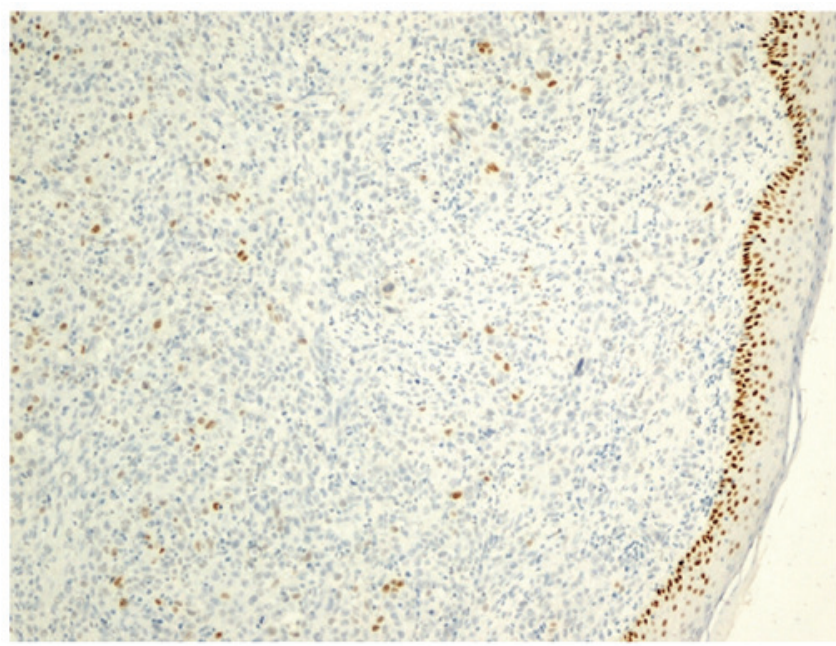

Figure 4. Immunohistochemical staining revealed positivity for focal p63 (stain, hematoxylin and eosin; magnification, x100).

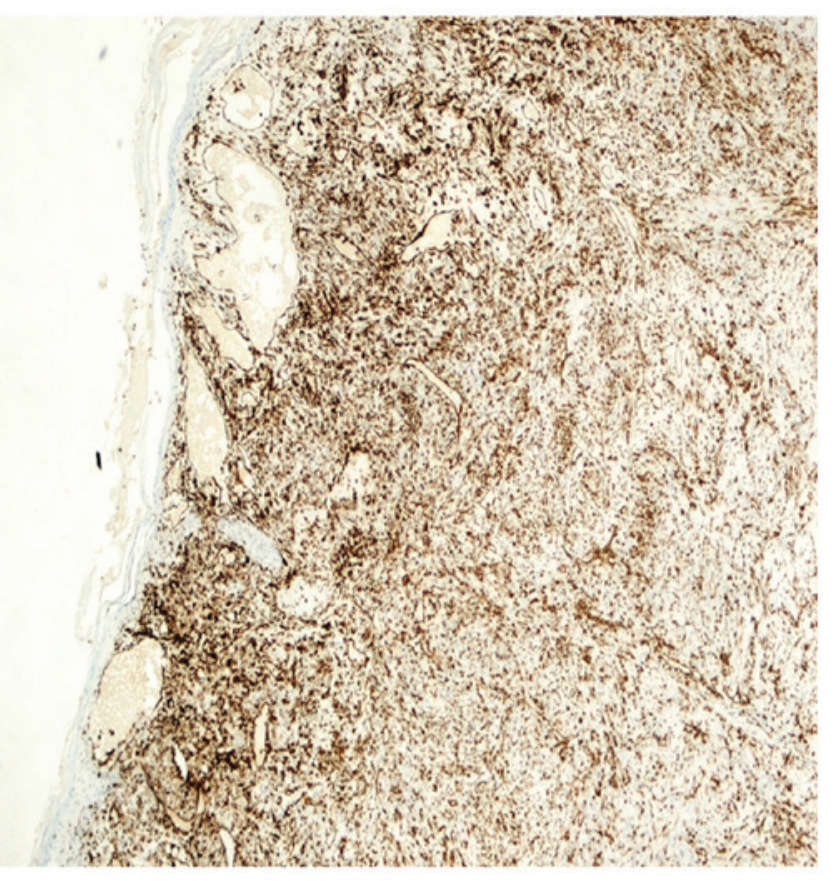

Figure 5. Immunohistochemical staining of tumoral tissue revealed positivity for vimentin (stain, hematoxylin and eosin; magnification, $\mathrm{x} 100$ ).

and embedded in paraffin blocks. The paraffin-embedded specimens were cut into $5 \mu \mathrm{m}$-sections and stained with hematoxylin and eosin for histological analysis under a light microscope. The histological analysis revealed pleomorphic mitotic cells with a large pale nucleus and coarse chromatin, as well as malignant epithelioid infiltration and the formation of vortex-like structures.

An immunohistochemical analysis involved staining the biospy sections with the following antibodies: Mouse anti-pan cytokeratin (CK) monoclonal mixture (1:300; cat. no. 313M-14; Cell Marque ${ }^{\mathrm{TM}}$; Sigma-Aldrich, Rocklin, CA, USA); mouse anti-epithelial membrane antigen (EMA) monoclonal antibody (1:200; cat. no. AC13038A,C; Biocare Medical, LLC., Concord, CA, USA); mouse anti-CK5/6 
monoclonal antibody (1:50; cat. no. 356M-14; Cell Marque ${ }^{\mathrm{TM}}$; Sigma-Aldrich); mouse anti-vimentin monoclonal antibody (1:400; cat. no. NCL-C-VIM572; Thermo Fisher Scientific, Inc.); rabbit anti-focal p63 monoclonal antibody (1:50; cat. no. BSB5852; Bio SB, Inc., Goleta, CA, USA); mouse anti-desmin monoclonal antibody (1:100; cat. no. 61-0077-2; Genemed Biotechnologies, Inc., South San Francisco, CA, USA); and mouse anti-cluster of differentiation 34 (CD34) monoclonal antibody (1:600; cat. no. MS-363-P0; Thermo Fisher Scientific, Inc., Waltham, MA, USA). Subsequently, the biopsy sections were incubated with horseradish peroxidase-conjugated rabbit anti-mouse and polymer anti-rabbit secondary antibodies (Bond ${ }^{\mathrm{TM}}$ Polymer Refine Detection; cat. no. DS9800; Leica Microsystems GmbH, Wetzlar, Germany), followed by 3,3'-diaminobenzidine tetrahydrochloride hydrate. The stained sections were observed under a light microscope. Staining revealed positivity for pan CK, EMA, CK5/6, vimentin and focal p63, and negativity for desmin and CD34. The patient was diagnosed with synchronous squamous cell carcinoma with sarcomatoid differentiation of the scalp (Figs. 2-5).

The patient was referred to the Department of Plastic Surgery (Sakarya University Training and Research Hospital) for resection; however, he refused treatment and was subsequently discharged to seek treatment at a different hospital in Ankara, Turkey. However, after 6 months, we were informed by a relative of the patient that he had succumbed to respiratory failure. Written informed consent was obtained from the patient for the publication of the present study.

\section{Discussion}

The prolongation of the human life span, as well as the continuous development of diagnostic and therapeutic strategies, have increased the incidence of secondary primary malignancies (7). According to Warren and Gate's criteria, synchronous tumors are diagnosed based on three factors: i) The diagnosis of malignancy for any tumor must be made conclusively; ii) each must be a different tumor; and iii) tumors must not result from the metastasis of another tumor (8). In patients with a malignancy, the occurrence of secondary malignancies may be coincidental or a multifactorial process (9). Smoking is an etiological risk factor for bladder, head, neck, lung and uterus cancer $(9,10)$. Following the diagnosis of a primary malignancy, patients who stop smoking significantly prolong the time it takes for a second primary malignancy to develop (11). Synchronous or metachronous tumors are rare in the urogenital system and may be associated with genetic disorders, including von Hippel Lindau disease, tuberous sclerosis and 3:8 chromosomal translocations (12). Stamey et al (13) reported that, in a previous study, prostate adenocarcinoma occurred in $40 \%$ of patients that underwent cystoprostatectomy for the treatment of bladder cancer. Furthermore, Pastore et al (14) reported the synchronous occurrence of transitional cell carcinoma of the urinary bladder, breast and primary neoplasms of the skin in one patient. The patient in the present case developed synchronous squamous cell carcinoma of the scalp 3 months subsequent to the diagnosis of papillary bladder cancer.

In conclusion, the present study supported the hypothesis that, in patients with a primary cancer, the possibility of a secondary primary malignancy in the same or a different organ is increased. Furthermore, if a secondary tumor is identified, the risk of relapse or metastasis must be considered. To the best of our knowledge, no cases of synchronous skin malignancy and urothelial carcinoma of the bladder have been reported previously.

\section{References}

1. Shikhani AH, Matanoski GM, Jones MM, Kashima HK and Johns ME: Multiple primary malignancies in head and neck cancer. Arch Otolaryngol Head Neck Surg 112: 1172-1179, 1986.

2. Martini N and Melamed MR: Multiple primary lung cancers. J Thorac Cardiovasc Surg 70: 606-612, 1975.

3. Duchateau CS and Stokkel MP: Second primary tumors involving non-small cell lung cancer: Prevalence and its influence on survival. Chest 127: 1152-1158, 2005.

4. Mydlo JH and Gerstein M: Patients with urologic cancer and other nonurologic malignancies: Analysis of a sample and review of the literature. Urology 58: 864-869, 2001.

5. An G, Ng AY, Meka CS, Lou G, Bright SP, Cazares L, Wright GL Jr. and Veltri RW: Cloning and characterization of UROC28, a novel gene overexpressed in prostate, bladder and breast cancers. Cancer Res 60: 7014-7020, 2000.

6. López ML, Lana A, Díaz S, Folgueras MV, Sánchez L, Comendador MA, Belyakova E, Rodríguez JM and Cueto A: Multiple primary cancer: An increasing health problem. Strategies for prevention in cancer survivors. Eur J Cancer Care (Engl) 18: 598-605, 2009.

7. Wallace D, Arul D and Chitale S: Synchronous tumours of the breast and bladder. J Surg Case Rep 2014: rju066, 2014.

8. Warren S and Gates O: Multiple primary malignant tumors; A survey of the literature and statistical study. Am J Cancer 16: 1358-1414, 1932.

9. van Bodegom PC, Wagenaar SS, Corrin B, Baak JP, Berkel J and Vanderschueren RG: Second primary lung cancer: Importance of long term follow up. Thorax 44: 788-793, 1989.

10. Antal A and Vallent K: Cases of multiple tumors in our clinic. Orv Hetil (abstract) 138: 1507-1510, 1997.

11. Ozseker F, Bilgin S, Baran A, Dilek İ, Bayram U and Akkaya E: Second Primary Lung Cancers. Solunum Hastalıklar1 14: 239-244, 2003 (In Turkish).

12. Conquy S, Steg A and Ferry M: Bilateral kidney cancer in a patient with Von Hippel-Lindau disease. Ann Urol (Paris) 21: 350-352, 1987 (In French).

13. Stamey TA, Freiha FS, McNeal JE, Redwine EA, Whittemore AS and Schmid HP: Localized prostate cancer. Relationship of tumor volume to clinical significance for treatment of prostate cancer. Cancer 71 (Suppl 3): S933-S938, 1993.

14. Pastore AL, Palleschi G, Autieri D, Leto A, Ripoli A, Maggioni C, Moschese D, Al Salhi Y, Porta N, Di Cristofano C, et al; Sapienza University of Rome, Faculty of Pharmacy and Medicine: Synchronous primary neoplasms of the bladder, skin and breast in a male patient: A case report. World J Surg Oncol 11: 282, 2013. 